\title{
Acute kidney injury: top ten tips
}

\author{
Alison M Prescott, Andrew Lewington and Donal O'Donoghue
}

\begin{abstract}
Acute kidney injury (AKI) is associated with increased patient morbidity and mortality, and represents a significant financial burden for the NHS. The National Confidential Enquiry into Patient Outcomes and Death (NCEPOD)'s report, Adding insult to injury, demonstrated that only $50 \%$ of patients who died from AKI received good care and $30 \%$ of patients had predictable and avoidable AKI. It is therefore essential to identify patients at risk of AKI early and to treat patients who develop it promptly. This article proposes how this may be achieved by highlighting ten top tips that describe the points along the patient pathway where it is possible to intervene and prevent or treat AKI. The tips emphasise the importance of good basic medical care and the need for engagement with patients, healthcare professionals and hospital processes. The implementation of these tips in hospitals across the UK could potentially improve patient outcomes and reduce associated costs.
\end{abstract}

KEY WORDS: acute kidney injury, acute renal failure, detection, management, prevention

\section{Introduction}

Acute kidney injury (AKI) is a condition in which there is rapid decline in kidney function over a period of hours to days, which results in a failure to regulate fluid, electrolyte and acid-base balance. It is a common and serious condition which has recently been demonstrated to be both predictable and avoidable in $20 \%$ of patients who develop AKI following admission and subsequently die. ${ }^{1}$ An improvement in patient outcomes with early recognition and treatment of AKI has been demonstrated by the military, where AKI is now virtually unheard of in conflict situations due to improved fluid resuscitation and acute care. ${ }^{2}$ This suggests that the implementation of appropriate systems within the NHS could similarly improve patient treatment and outcomes. This article proposes ten top tips that highlight the areas along the patient pathway - from risk in the community for AKI through to developing severe AKI - in which it may be possible to intervene and reduce both morbidity and mortality.

Alison M Prescott, ${ }^{1}$ foundation year 1 trainee in acute medicine; Andrew Lewington, ${ }^{1}$ consultant renal physician/honorary senior lecturer; Donal O'Donoghue, ${ }^{2}$ national clinical director for kidney care and consultant renal physician

1St James's University Hospital, Leeds; ${ }^{2}$ Salford Royal NHS Foundation Trust, Manchester

\section{Definition and staging of AKI}

The concept of 'acute renal failure', in which there is failure of the kidney filtration function requiring treatment with dialysis, was introduced in the mid 1940s. This has subsequently been replaced by the term AKI to better represent the different degrees of kidney injury that occur before kidney function fails: from its early onset as injury, to progressive loss of kidney function and finally to kidney failure requiring renal replacement therapy (RRT). More recently, even small increases in serum creatinine have been recognised to be associated with worse patient outcomes. Newer definitions for AKI have reflected this increased awareness and have incorporated these relatively small increases in serum creatinine. The adoption of a universal definition for AKI by the international guideline group Kidney Diseases: Improving Global Outcomes (KDIGO) is anticipated to promote earlier recognition and treatment of the condition, and translate clinically into reduced kidney injury. ${ }^{3,4}$ The KDIGO group defines AKI as fulfilment of one of the following criteria: $^{5}$

- increase in serum creatinine $\geqslant 26 \mu \mathrm{mol} / \mathrm{L}$ from baseline value within 48 hours

- increase in serum creatinine $\geqslant 1.5$ times the baseline value within one week

- urine output $<0.5 \mathrm{ml} / \mathrm{kg} /$ hour for more than six consecutive hours.

Patients diagnosed with AKI can be staged using the KDIGO staging system (Table 1). ${ }^{5}$ Patients are staged according to whichever criteria (creatinine, urine output or receipt of RRT) gives them the highest stage for that episode of AKI. Studies have

Table 1. Kidney Disease: Improving Global Outcomes (KDIGO) staging system for acute kidney injury. ${ }^{5}$

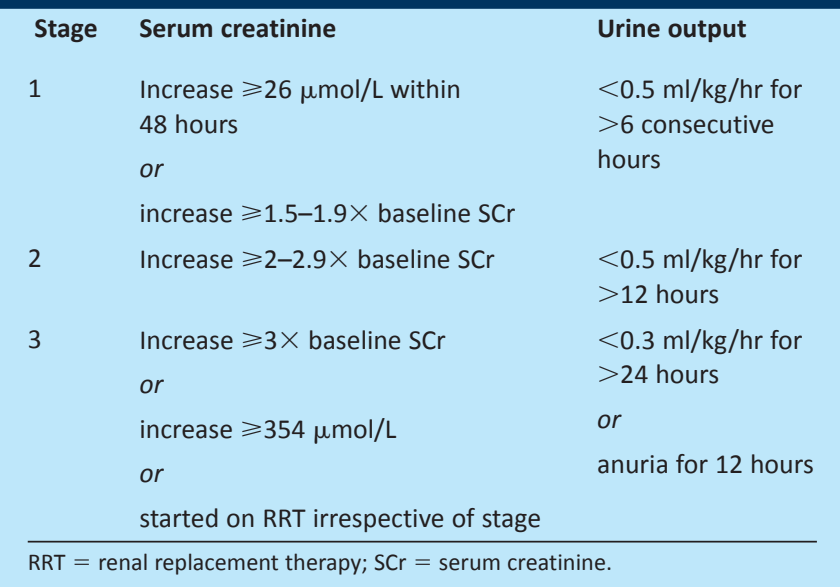


demonstrated that sharp increases in mortality are associated with increasing stages of AKI. ${ }^{6}$

When a patient is diagnosed with AKI, it is essential to identify the underlying cause. The aetiology of AKI is divided into three categories: pre-renal, intrinsic renal and post-renal (Table 2). Although studies have shown that these account for $55-60 \%$, $35-40 \%$ and $<5 \%$ of cases of AKI, respectively, most cases are multifactorial, with $75 \%$ of cases being secondary to a combination of pre-renal failure (hypoperfusion) and intrinsic acute tubular injury (hypoperfusion, sepsis and nephrotoxins). ${ }^{7,8}$

\section{Epidemiology}

Acute kidney injury is a significant cause of morbidity and mortality, particularly among more elderly patients with multiple comorbidities, and represents a substantial financial burden to the NHS. The previous absence of a universal definition for AKI has meant that its true incidence is uncertain. Recent population studies based in the UK have estimated an annual incidence of 486-620 per million population, depending on definition, which accounts for about $1 \%$ of hospital admissions and complicates more than $7 \%$ of hospital inpatient stays. ${ }^{10,11}$

Estimates indicate that $5-10 \%$ of critically ill patients will develop AKI. The prevalence of receipt of RRT as a result of AKI is $22-203$ per million population, with up to $49 \%$ of those admitted to the intensive care unit (ICU) needing renal support. ${ }^{12}$ Acute kidney injury accounts for $10 \%$ of all bed-days in ICU and is associated with prolonged inpatient stays. It is estimated to cost the NHS between $£ 434$ million and $£ 640$ million per year. ${ }^{7,13}$ A recent study in the UK reported an overall inpatient mortality of $23.8 \%$, ranging from $16.1 \%$ for stage $1 \mathrm{AKI}$ to $36.1 \%$ for stage 3 . Renal recovery was dependent on the severity of AKI, with $80.0 \%$ recovery in stage 1 falling to $58.8 \%$ for stage $3 .{ }^{14}$

\section{Top ten tips for the prevention, identification and management of AKI}

All hospital patients are at risk of AKI - either through their presenting illness or subsequent iatrogenic injury. With up to $30 \%$ of deaths from AKI being preventable through early recognition and management of patient risk factors, it is essential for all clinicians to have a thorough knowledge of the risk factors that predispose patients to AKI (Box 1). ${ }^{1}$ Patients with one or more risk factors should undergo frequent biochemical monitoring and clinical review of fluid balance, volume status and medications. ${ }^{15}$

Clinicians often view the management of AKI as challenging because of its perceived complexity. However, studies have demonstrated that, all too often, even the basics in care (such as fluid balance, intravenous fluids and regular blood monitoring) are neglected. ${ }^{16}$ This was highlighted in the National Confidential Enquiry into Patient Outcome and Death (NCEPOD) report on AKI in 2009, in which only $50 \%$ of patients were deemed to have received a 'good' standard of care. ${ }^{1}$ This article proposes ten top
Table 2. Major causes of acute kidney Injury. ${ }^{9}$

\begin{tabular}{|c|c|c|}
\hline Classification & Causes & \\
\hline \multirow[t]{3}{*}{ Pre-renal } & Hypovolaemia & $\begin{array}{l}\text { Haemorrhage } \\
\text { Gastrointestinal losses } \\
\text { Renal losses } \\
\text { Burns }\end{array}$ \\
\hline & $\begin{array}{l}\text { Reduced cardiac } \\
\text { output }\end{array}$ & $\begin{array}{l}\text { Cardiac failure } \\
\text { Liver failure } \\
\text { Sepsis } \\
\text { Drugs } \\
\text { ACE inhibitor }\end{array}$ \\
\hline & Drugs & NSAIDs \\
\hline \multirow[t]{3}{*}{ Renal } & Vascular & $\begin{array}{l}\text { Vasculitis } \\
\text { Thrombosis } \\
\text { Emboli } \\
\text { Dissection }\end{array}$ \\
\hline & Glomerular & Glomerulonephritis \\
\hline & $\begin{array}{l}\text { Tubular } \\
\text { Interstitial }\end{array}$ & $\begin{array}{l}\text { Ischaemia } \\
\text { Rhabdomyolysis } \\
\text { Myeloma } \\
\text { Nephrotoxic medication } \\
\text { Interstitial nephritis } \\
\text { Lymphoma }\end{array}$ \\
\hline Post-renal & Obstruction & $\begin{array}{l}\text { Kidney stones } \\
\text { Catheter } \\
\text { Enlarged prostate } \\
\text { Pelvic mass } \\
\text { retroperitoneal fibrosis }\end{array}$ \\
\hline
\end{tabular}

Box 1. Common risk factors for acute kidney injury. ${ }^{16}$

1 CKD (eGFR $<60 \mathrm{ml} /$ minute $\left./ 1.73 \mathrm{~m}^{2}\right)$

2 Age $>75$ years

3 Atherosclerotic peripheral vascular disease

4 Cardiac failure

5 Liver disease

6 Diabetes mellitus

7 Nephrotoxic drugs, eg

- NSAIDs

- Aminoglycosides

8 Hypotension (compared to baseline blood pressure)

- Hypovolaemia

- Primary cardiac cause

- Antihypertensive medication

9 Sepsis

CKD = chronic kidney disease; eGFR = estimated glomerular filtration rate; NSAID $=$ non-steroidal anti-inflammatory drug. 


\section{Box 2. Top ten tips for the prevention, identification and management of acute kidney injury (AKI).}

1 Sick-day rules

2 Trainee induction programmes

3 Hospital policies

4 12-hour senior review

5 Electronic alerts

6 AKI guidelines

7 AKI patient pathways

8 Good handover to prevent inhospital AKI

9 Coding and audit of pathways, transfers and delays

10 Patient follow-up

tips for the prevention, identification and management of AKI in a hospital setting, for use by all clinicians regardless of specialty or grade (Box 2).

\section{Tip 1: sick-day rules}

Patients with risk factors (see Fig 1) should be warned of the possibility of developing AKI if they become acutely ill, especially with diarrhoea and vomiting. They should be advised to increase their fluid intake and avoid any nephrotoxic medication while ill. Empowering patients to take charge of their own healthcare could prevent a number of cases of AKI that develop in the community.

\section{Tip 2: trainee induction programmes}

Acute kidney injury is a common medical emergency encountered across all medical specialties. The NCEPOD report in 2009 demonstrated that poor management of AKI was most commonly the result of poor clinical care rather than organisational issues. It is therefore essential that all doctors, regardless of grade and specialty, should receive regular teaching on AKI. ${ }^{1}$ It is recommended that this occurs at trust induction for foundation year trainees and subsequently for core medical, general practitioner (GP) and specialty trainees. Such teaching could be delivered through lectures, tutorials, simulation-based learning or elearning packages such as the AKI module provided by e-Learning for Healthcare. ${ }^{17}$

\section{Tip 3: hospital policies}

The National Institute for Health and Clinical Excellence (NICE) recommends the use of physiological track-and-trigger systems, such as the National Early Warning System (NEWS), in all hospitals to enable acutely ill patients to be identified and monitored. ${ }^{18}$ This system ensures that patients' physiological observations are recorded at least every 12 hours, with increased frequency in those with abnormal physiology.

All staff who care for patients in acute hospital settings should have competencies in monitoring, interpretation of results and provision of prompt response to acutely ill patients appropriate to the level of care that they provide. ${ }^{19}$ Such a competency framework for AKI has been published by the Academy of Royal Colleges. Trusts should provide education and training in these competencies and carry out regular assessments to ensure that all staff can demonstrate them. ${ }^{19}$ With regard to AKI, nursing staff must be competent in recording of fluid balance and doctors in assessing volume status, interpreting fluid-balance charts and prescribing appropriate intravenous fluids.

\section{Tip 4: 12-hour senior review}

The NCEPOD report highlighted a correlation between the grade of doctor carrying out the initial patient assessment and the overall quality of the patient's care. ${ }^{1}$ A higher proportion of patients admitted by senior-grade doctors had hospital stays in which the overall quality of care was deemed to be 'good' than those admitted by junior doctors. The report also showed that although fewer patients develop AKI as inpatients than are admitted with the condition ( $12 \%$ and $18 \%$ respectively), the quality of care for those developing AKI while an inpatient is poorer than for those diagnosed on admission (33\% compared to $50 \%$ receiving 'good' quality care respectively). This difference was due in part to delayed recognition of those who develop AKI after admission, with $74 \%$ of patients having stage 2 or 3 AKI by the time the condition was recognised. ${ }^{1}$ It is therefore recommended (in accordance with the Royal College of Physicians' policy) that all patients should have a consultant review within 12 hours of admission. ${ }^{1}$ A senior review will promote improved quality care and should ensure that patients with risk factors for developing AKI during their hospital stay (see Fig 1) are identified and suitable management plans put in place.

\section{Tip 5: electronic alerts}

The NCEPOD report found deficiencies in care in more than $50 \%$ of patients, many of which resulted from delays in diagnosis as a result of failure to carry out or act upon abnormal results for routine biochemistry monitoring and urinalysis. ${ }^{1}$ As a result, the report recommended the use of electronic alert systems to ensure that abnormal serum creatinine results are acted upon. Studies looking at the efficacy of the systems currently in use have shown a 55\% reduction in the risk of AKI in patients taking nephrotoxic drugs and an improvement in medicines management in $52 \% .20,21$ More recently, Selby et al described a real-time electronic resultsreporting system for AKI, which is linked to clinical guidelines on the hospital intranet. This system not only improved early recognition of AKI but also encouraged timely management of patients and early escalation of sick patients in accordance with local guidelines. ${ }^{14}$

\section{Tip 6: AKI guidelines}

Although the management of patients diagnosed with AKI is ultimately dependent on the underlying aetiology, initial supportive management should include clinical assessment of volume 
status, appropriate fluid resuscitation and detailed medication review. Nephrotoxic medications should be stopped and appropriate dose adjustments made for medications metabolised and/ or excreted by the kidneys. With studies demonstrating that even these basics are neglected, clear guidelines should be available for the initial management of patients with AKI. ${ }^{1}$

Guidelines for the management of AKI are due to be published by NICE in 2013; until then, clinicians should refer to the guidelines published by KDIGO and the Renal Association. ${ }^{5,13}$ National guidelines should be used to influence local guidelines, which can be adapted to local systems (early warning systems and electronic alert systems) and in keeping with local referral criteria, to enable early identification and escalation of sick patients.

\section{Tip 7: AKI patient pathways}

Although all clinicians should be capable of managing cases of mild AKI, clear patient pathways should be in place to enable rapid escalation of severe or complicated cases to specialised renal teams. The NCEPOD report found that $20 \%$ of patients who required referral to the renal team were not referred and $21 \%$ of the referrals made were considered to be delayed, possibly as a result of the ward team failing to recognise the severity of AKI. As such, the report recommended that defined referral criteria and/or transfer protocols should be put in place in all hospitals to ensure that every patient with severe or complicated AKI is referred for renal review. ${ }^{1}$ The Royal College of Physicians has accredited the Map of Medicine AKI pathway which includes management and referral criteria for patients in both primary and secondary care. ${ }^{22}$

\section{Tip 8: good handover to prevent inhospital AKI}

The NCEPOD report found that $14 \%$ of the reviewed cases of AKI had been avoidable, which correlates with the results of other studies in which volume depletion during acute illness and use of nephrotoxic medication were identified as the main preventable causes. ${ }^{1,17}$ Given the poor outcomes associated with AKI, it is essential that steps are taken in hospitals to prevent AKI.

Following identification of patients at risk of developing AKI and implementation of appropriate management plans through 12-hour senior reviews, good handover between acute and base wards is essential to ensure continuity of patient care. With the involvement of both nursing staff and medical staff in the monitoring of patients at risk of AKI, it is essential that these patients are highlighted to both teams. Clear documentation of patients' monitoring is also a necessity. One possible approach to this challenge would be a single pro forma, on which the results of urinalysis, fluid balance and biochemistry are recorded, for both nursing and medical staff to use to monitor patients at risk of AKI. This would be kept in the patient's observations folder, where it could be updated daily and reviewed on ward rounds. The pro forma would enable any deterioration in the patient's urine output or serum creatinine to be identified quickly. Electronic systems such as VitalPAC, which provide the same functions, are available in some hospitals.

\section{Tip 9: coding and audit of pathways, transfers and delays}

Regular audits of the identification of and care received by patients with AKI are essential to determine standards of care locally. The results should highlight areas of weakness and guide the development of local protocols to improve patient care. The involvement of clinicians from different specialties in the audit cycle should be encouraged to raise awareness of AKI in all medical and surgical specialties.

Correct coding of episodes of AKI using the International statistical classification of diseases and health-related problems, tenth edition, is essential to ensure that accurate statistical data on the condition is collected to allow hospital trusts to set aside appropriate resources to manage the condition. Accurate coding also ensures correct payment for the trust, as coded clinical data is grouped to meet the reporting structure of payment by results. The importance of coding should be discussed at trust induction for junior doctors, so that their role in clearly documenting the diagnosis of AKI in the medical notes, to enable correct coding and dictate future management, is highlighted.

\section{Tip 10: patient follow up}

Following an episode of AKI, patients are at risk of developing chronic kidney disease (CKD). The risk is long term and increases with increasing severity of AKI. ${ }^{6}$ Prior to discharge, patients should have a full medication review and a plan should be made to reintroduce carefully any medications that were withheld during the acute illness. The patient's episode of AKI must be documented clearly on their discharge note, even if it was not the primary diagnosis. This is essential to alert the patient's GP to the increased risk of further episodes of AKI, particularly with the use of nephrotoxic medications, and the increased risk of CKD. The GP should also be informed of the plan to reintroduce withheld medications and should be asked to check the patient's kidney function shortly after discharge and to register those with evidence of CKD on the primary care CKD register.

It is recommended that patients are informed why they developed AKI and how they can try to avoid further episodes. Patient education leaflets on AKI should be provided so that patients can remind themselves of this information when necessary.

\section{Conclusion}

Acute kidney injury is not a specialist's emergency; it is seen commonly in acute medicine and, as such, it is essential that all physicians have the confidence and skills to identify and manage it. The NCEPOD report in 2009 found that poor management of AKI was most commonly due to poor clinical care rather than organisational issues, with the basics of medical care being 
omitted from patient management plans. However, organisational and systematic approaches can prompt and assist with care of AKI. The top ten tips proposed aim to emphasise the importance of good basic medical care while recognising that the prevention and management of AKI requires involvement at every level: by the patient (patient education), by healthcare professionals (education of junior doctors, nurses and pharmacists, and implementation of senior review and guidelines) and by hospitals (through electronic alerts, access to services and audit). Implementing the top tips in individual practices and across acute hospitals could reduce the mortality and morbidity from AKI and its financial burden on the NHS.

\section{References}

1 National Confidential Enquiry into Patient Outcomes and Death. Adding insult to injury - a review of care of patients who died in hospital with a primary diagnosis of acute kidney injury (acute renal failure). London: NCEPOD, 2009. www.ncepod.org.uk/2009aki.htm [Accessed 31 May 2012].

2 World MJ. Military nephrology - what a civilian doctor should know. Nephrol Dial Transpl 2011;4:153-7.

3 Kidney Disease: Improving Global Outcomes. Kidney Disease: Improving Global Outcomes (KDIGO). New York: KDIGO, 2008. www. kdigo.org/clinical_practice_guidelines_3.php [Accessed 31 May 2012].

4 Lassnigg A, Schmidlin D, Mouhieddine M et al. Minimum changes of serum creatinine predict prognosis in patients after cardiothoracic surgery: a prospective cohort study. J Am Soc Nephrol 2004;15:1597.

5 Kidney Disease Improving Global Outcomes. KDIGO clinical practice guideline for acute kidney injury. Kidney Int 2012;2:1-140.

6 Ahistrom A, Kuitunen A, Peltonen S et al. Comparison of 2 acute renal failure severity scores to general scoring systems in the critically ill. Am J Kidney Dis 2006;48:262-8.

7 Lameire N, Van Biesen W, Vanholder R. Acute kidney injury. Lancet 2008;372:1863-5.

8 Hou SH, Bushinsky DA, Wish JB et al. Hospital acquired renal insufficiency: a prospective study. Am J Med 1983;74:243.

9 Lines S, Lewington A. Acute kidney injury. Clin Med 2009;9:273-7.

10 Hegarty J, Middleton RJ, Krebs $\mathrm{M}$ et al. Severe acute renal failure in adults: place of care, incidence, and outcomes. QJM 2005;98:661-6.
11 Nash K, Hafeez A, Hou S. Hospital acquired renal insufficiency. Am J Kidney Dis 2002;39:930-6.

12 Lewington A, Kanagasundaram S. Renal association clinical practice guidelines on acute kidney injury. Nephron Clin Pract 2011;118(Suppl 1):c349-90.

13 Calculating the cost. Health Serv J 2011;121(Suppl):S1.

14 Selby NM, Crowley L, Fluck RJ et al. Use of electronic results reporting to diagnose and monitor AKI in hospitalized patients. Clin J Am Soc Nephrol 2012;7:533-40.

15 Griffiths L, Kanagasundaram NS. Assessment and initial management of acute kidney injury. Medicine 2011;39:390-7.

16 Stevens PE, Tamimi NA, Al-Hasani MK et al. Non-specialist management of acute renal failure. QJM 2001;94:533-40.

17 e-Learning for Healthcare. e-Learning for renal medicine. London: e-Learning for Healthcare, 2011. www.e-lfh.org.uk/projects/kidn-e/ index.html [Accessed 31 May 2012].

18 National Institute for Health and Clinical Excellence. Acutely ill patients in hospital: Recognition of and response to acute illness in adults in hospital. Clinical guideline 50. London: NICE, 2007. www.nice.org. uk/CG50 [Accessed 31 May 2012].

19 Finlay S. Acute kidney injury: a competency framework. Defining the role of the clinician. London: Academy of Medical Royal Colleges, 2011. www.aomrc.org.uk/publications/reports-a-guidance/doc_ download/9423-acute-kidney-injury-a-competency-framework.html [Accessed 31 May 2012].

20 Rind DM, Safran C, Phillips RS et al. Effect of computer-based alerts on the treatment and outcomes of hospitalized patients. Arch Intern Med 1994;154:1511-7.

21 McCoy AB, Waitman LR, Gadd CS et al. A computerized provider order entry intervention for medication safety during acute kidney injury: a quality improvement report. Am J Kidney Dis 2010;56:832-41.

22 Map of Medicine, 2012. http://app.mapofmedicine.com/mom/58/ login_page.html?next=http\%3A\%2F\%2Fapp.mapofmedicine. com\%2Fmom\%2F58\%2Fpage.html\%3Fdepartmentid\%3D4\%26specialty-id\%3D1014\%26pathway-id\%3D11185\%26pageid\%3D14054\%26history\%3Dclear [Accessed 2 July 2012].

Address for correspondence: Dr Andrew Lewington, Renal Department, Lincoln Wing, St James's University Hospital, Beckett Street, Leeds, West Yorkshire, LS9 7TF. Email: Andrew.Lewington@leedsth.nhs.uk Follow the author on Twitter: @ajpl1901 